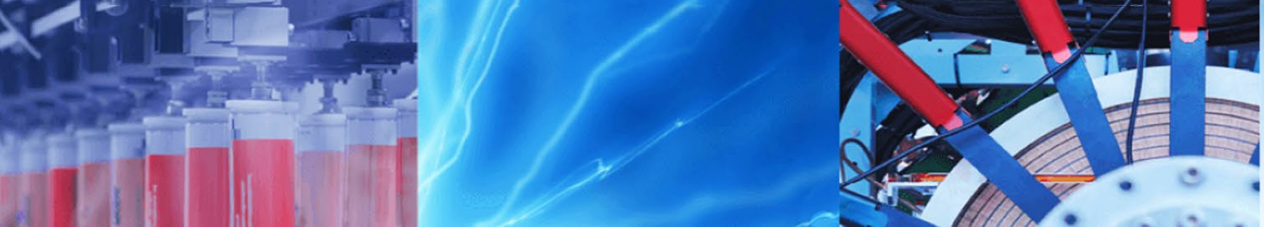

Research Article

\title{
Comparative spatial-temporal analysis and predictive modeling of climate change-induced malaria vectors' invasion in new hotspots in Kenya
}

\section{J. S. Kimuyu' ${ }^{1}$}

Received: 12 October 2020 / Accepted: 5 July 2021

Published online: 17 July 2021

(c) The Author(s) $2021 \quad$ OPEN

\begin{abstract}
Climate change/variability is a major driving factor among others that contribute to the spread of suitable malaria vectors' geographical extent. The current study employed comparative spatial-temporal analysis using bioclimatic envelope modeling to predict and quantify the possible surge of suitable malaria vectors' habitats in new hotspots in Kenya. BIOCLIM and BIOCLIM True/False models were run with model data from HADCM3, CCCMA and SCIRO IPCC future climatic projections under A2a scenario. Prediction and projection of the malaria vectors' prevalence and distribution were done for the whole country. Spatial-temporal models were generated for the baseline climate, and projections were done to depict how the vectors are likely to be distributed by the years 2020, 2050 and 2080 under the influence of climate change. The results showed that the highest suitable malaria vectors' habitats by area was $227,092 \mathrm{~km}^{2}$ obtained when prediction was done with HADCM3 future climate by the year 2050. The least suitable habitats by area was $80,060 \mathrm{~km}^{2}$ which was predicted with CCCMA projection by the year 2050. Ecological niche prediction from HADCM3 and CSIRO showed a similar trend although at different magnitudes. The prediction results portrayed high likelihood of shift in some suitable habitats that could turn unsuitable, while new hotspots are likely to emerge. The BIOCLIM prediction with the three future climate models showed that the current endemic zones of the lake region and south coastal strip of the Indian Ocean will still remain suitable habitats but with a decline shift in spatial extent by the year 2020, then start to expand by the years 2050-2080. Predictions from HADCM3 by the year 2050 has shown possible wide spread of malaria spatial extents in counties like Narok, Kajiado, Kitui, Makueni, Machakos, Meru, Marsabit, Isiolo, Samburu, Baringo, West Pokot, Turkana and Mandera, while a few others of lower extent might have some emerging isolated hotspots. Laikipia County might become unsuitable habitat for malaria vectors by the year 2050 , and the case may remain the same by the year 2080. The malaria burden is likely to shift from Laikipia to the neighboring counties of Baringo, Isiolo, Meru and Turkana. In conclusion, malaria vectors are likely to spread in new continuous and isolated hotspots with future likely increase in malaria prevalence hence possible epidemic upsurge by the year 2050. Consequently, evidence-based scientific research can be utilized to guide policy for sustainable development in the health agenda.
\end{abstract}

Keywords Spatial-temporal analysis · Bioclimatic envelope modeling · Malaria vectors $\cdot$ Habitats · Ecological niche modeling (ENM) - Malaria prevalence

J. S. Kimuyu, kimuyu.jacinta@gmail.com | 'Department of Geography and Spatial Systems, Technical University of Kenya, Nairobi, Kenya.

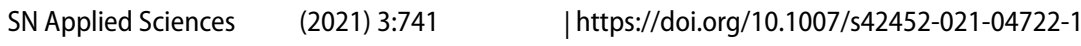




\section{Introduction}

Malaria, which is a vector-borne disease, still remains a major public health concern with great socioeconomic impact on the population living in endemic zones in Kenya. Climate change has potential to enhance the vectors' distribution significantly with possible emergence of the disease in new regions. Currently, malaria cases have been reported in new counties and certain parts of the country that were non-endemic zones previously. Ecological niche modeling (ENM) has demonstrated significant alteration in suitability of malaria vectors' habitats in Kenya from the current ecological zones by the years 2020, 2050 and 2080 [1]. Earlier studies were done in Kenya to predict the burden of disease [2], map the risk of malaria infection [3], model the global constraints of temperature on transmission of Plasmodium falciparum and P. vivax [4] and generate spatial and temporal malaria risk maps that are essential tools to monitor the impact of control, evaluate priority areas to reorient intervention approaches and investments in malaria endemic counties [5]. In the current study, spatial-temporal models were generated for the baseline climate, and projections were done to depict how the vectors are likely to be distributed by the years 2020, 2050 and 2080 under the influence of climate change/variability. Predictions from HADCM3 by the year 2050 has shown possible wide spread of malaria spatial extents in counties like Narok, Kajiado, Kitui, Makueni, Machakos, Meru, Marsabit, Isiolo, Samburu, Baringo, West Pokot, Turkana and Mandera, with a few other counties of lower extent that might have some emerging isolated malaria hotspots. Laikipia County might become unsuitable habitat for malaria vectors by the year 2050 and the case remains the same by the year 2080 . The malaria burden is likely to shift from Laikipia to the neighboring counties of Baringo, Isiolo, Meru and Turkana; thus, early preparedness is warranted.

Spatial models provide visualization tools for evidence-based decision support to guide strategic policy on malaria eradication interventions. The comparative spatial-temporal study aims to link environmental variables with malaria vectors' presence through predictive modeling to delineate possible future suitable hotspots for the vectors' invasion hence malaria prevalence. The research study employed BIOCLIM and BIOCLIM True/ False models to determine and compare model outputs of the possible future malaria vectors' hotspots by ecological niches. Interventions targeting the hotspots may accelerate reductions in transmission and could be pivotal for malaria elimination [6]. Effective vector control strategy requires information on malaria vectors' prediction and distribution; thus, the research in this area has been of significant interest globally [7-13]. Public health interventions require the crucial information on malaria occurrence and distribution patterns with quantified spatial-temporal extent of the underlying suitable habitats for the malaria vectors' to thrive. Malaria surveillance and eradication strategy should leverage on visualization models in space and time to identify projected possible malaria hotspots toward development of disease preparedness strategy.

The timely study is well-justified since malaria has persisted as a major cause of morbidity and mortality in Kenya with more than 70 percent of the population at risk of the disease [14]. The indigenous communities have continued to be subjected to the deteriorating physical conditions of their particular ecological habitats and the natural catastrophes emanating from climate change/variability over decades. A world free of malaria is a major goal of global health, unequivocally embraced by the World Health Organization (WHO) soon after its founding in 1948 [15]. Managing the risks of climate change involves adaptation and mitigation decisions with implications for future generations, economies, and environments [16].

\section{Methodology}

The spatial-temporal distributions of the main malaria vectors in Kenya were quantified using BIOCLIM and BIOCLIM True/False models to determine the relationship between vector distribution and climate change/ variability. The methodology employed in this research was ecological niche modeling (ENM), a technique that encompasses a suite of tools that relate the known occurrences of malaria vectors to raster geographic information system layers that summarize variation in several environmental dimensions. There exist varieties of environmental modeling approaches that are capable of being applied to generate species distribution in baseline and future ecologies under climate change scenarios. The selection of appropriate modeling methodology largely depends on the type of data available. Correlative species distribution models require two types of data input; biological data, describing the known species distribution and environmental data, describing the landscape in which the species is found. This study selected BIOCLIM and BIOCLIM True/ False models as they are useful in modeling presence only malaria vector distribution data, and prediction is made without any reference to other samples in the study area.

Rendering exploited geospatial techniques and geostatistical modeling software to generate climate envelope prediction models from the baseline climate and future projections. The intention was to depict the vectors' likely 
future distribution in space and time by the years 2020, 2050 and 2080 under different climate change scenarios. The two approaches that can be applied to characterize environmental conditions that are suitable for species are either mechanistic or correlative. When mechanistic models are used, they incorporate physiologically limiting mechanisms in a species' tolerance to environmental conditions. On the other hand, use of correlative models aims to estimate the suitable environmental conditions for a species by associating known species' occurrence records with suites of environmental variables that can reasonably be expected to affect the species' physiology and probability of persistence. This study embarked on correlative approach to estimate the future suitable malaria vectors' ecologies as impacted by climate change.

\subsection{Data}

The malaria vector data acquired for this study was as amassed by Okara et al. [17], being primary empirical data from published and unpublished sources. The geolocated malaria presence data was collated for the period from 1990 to 2009. BIOCLIM and BIOCLIM True/False models were appropriately applied in modeling presence only malaria vector distribution data, and prediction was accomplished with DIVA-GIS freeware software. The environmental data was acquired and adopted as developed by Hijmans et al. 2005 [18] that comprised of the interpolated climate surfaces for global land areas, at a spatial resolution of 30 arc seconds (1-km spatial resolution). The climate elements included monthly precipitation and mean, minimum, and maximum temperature. After acquisition of the global climate surfaces from WorldCLIM database, further processing was done to extract by mask the national ESRI grid rasters for Kenya. The initial processing was done in ESRI ArcGIS software, then DIVA-GIS software was used to perform the predictions.

\subsection{Model selection}

In order to model the spatial-temporal distribution of malaria vectors in Kenya, the model-building process entailed model selection, model fitting, and model validation. These three basic steps are applied iteratively until an appropriate model for the data to be analyzed has been developed. The steps of model-building process are shown below (Fig. 1).

In the model selection step, plots of the data, process knowledge and assumptions about the process are used to determine the form of the model that can be fit to the data. Further to model selection, using the selected model and possibly information about the data, an appropriate model-fitting method is used to estimate the unknown parameters in the model. When the parameter estimates have been made, the model is then carefully assessed. The underlying assumptions of the analysis are qualitatively quantified for plausibility. Validity of the assumptions renders the model useful so as to answer the scientific questions that prompted the modeling effort. In case the model validation identifies problems with the current model, the modeling process is repeated using information from the model validation step to select and/or fit an improved model.

Bioclimatic variables were generated from three different future climate models, namely HADCM3, CCCMA and CSIRO all of 30 arc seconds resolution, under A2a scenario. The variables were generated in DIVA-GIS software, eventually used to run the ENM. The default coding for the bioclimatic variables is shown in Table 1. Ecological suitability prediction was based on the assumptions that malaria vector presence is restricted to locations equally or more suitable than those at which the species has been observed; and most environmental variables are continuous.

\subsection{Prediction and mapping of malaria vectors' ecological niches}

The two types of model input data used were known malaria vectors' occurrence records, and a suite of environmental variables. Raw environmental variables, such as precipitation and temperature records collected from weather stations, had been processed to generate model inputs [18]. The model inputs were processed in ArcGIS software and further used in DIVA-GIS software to produce bioclimatic variables that were thought to have a direct physiological role in limiting the ability of the species to survive. The principal steps executed in order to build and validate a correlative species' distribution model for malaria vectors in Kenya are given in Fig. 2 .

Ecological niche modeling was done with baseline climate data (1950-2000) and IPCC projected data for three climate models (HADCM3, CCCMA and CSIRO). The projected data was at 30-year interval, and predictive modeling was done and mapped for visualization and spatial analysis. ENM was performed with each data set to predict suitability habitats of malaria vectors and spatial models produced using BIOCLIM and BIOCLIM True/False predictions. The suitability maps for malaria vector prevalence were generated for visualization and arranged in succession from baseline climate (1950-2000), followed by the predicted prevalence by the year 2020, then 2050 and finally 2080 predictions for HADCM3, CCCMA and CSIRO, respectively. Discriminant function analysis (DFA) was applied to map different classes of suitable areas for malaria vectors to thrive using DIVA-GIS software. Areas completely outside the 
Fig. 1 Flow diagram showing the steps required in building a species distribution model

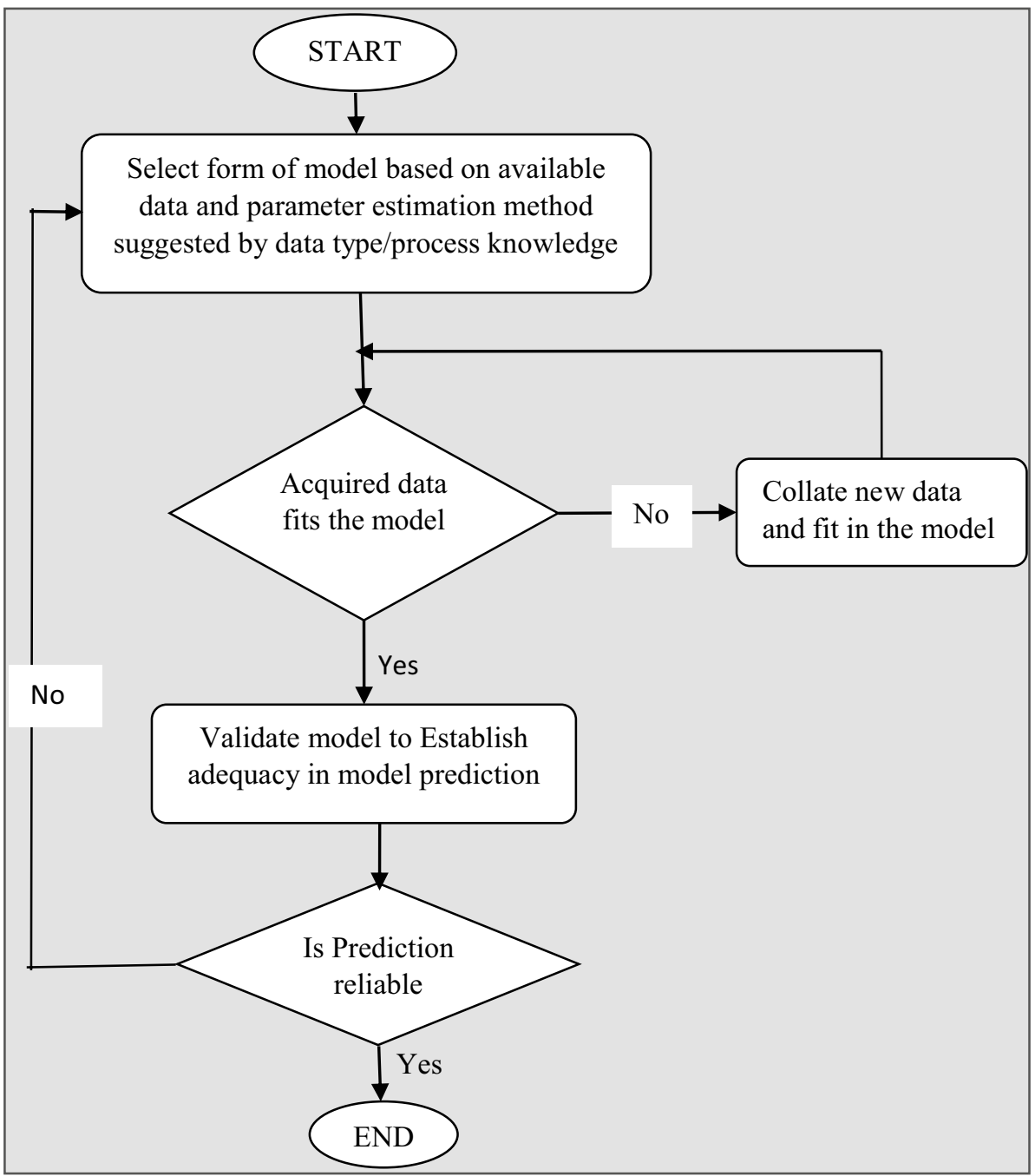

0-100 percentile envelope for one or more climate variables get a code " 0 ." The cells within the 5-95 percentile get a code " 3 ," those outside this range but within the 2.5-97.5 percentile get a code " 2 ," and the ones outside this but within the $0-100$ percentile for all climate variables get a code" 1 ." Different color shades were used to denote the suitability classes. The approach therefore identifies the minimum areas in which malaria vectors occurs while ensuring that no localities at which the vectors have been observed are omitted (i.e., omission rate $=0$ and sensitivity $=1$ ). Malaria vector distribution modeling was also done using BIOCLIM True/False species distribution model. All areas that fell within the envelope described by malaria presence sample points were cut off beyond a certain user-defined percentile and mapped using binary categorization as "true" (1) and "false" (0). All the areas that are found to be suitable for malaria vectors to thrive were denoted with red color while, those areas with no potential to sustain malaria vectors were shown in gray.

\subsection{Model validation}

The study employed predictive models and baseline data to project the possible emergence of malaria hotspots in areas that are non-endemic due to ecological shift emanating from climate change. The baseline malaria vector presence only data used was randomly divided into training and testing subsets with $75 \%$ of the records used in model training, while $25 \%$ of the records were used to test the model. This was achieved using DIVA-GIS software. The values provided is a measure of model performance in prediction by 2020,2050 and 2080. Random absence data was included from the mask since the validation requires an input of the presence-absence records. Point values were extracted from the generated bioclimatic grids and receiver operating characteristic (ROC) files from the presence-absence distribution of malaria vectors generated for the entire mask. Area under curve (AUC) and kappa statistic values were computed using the ROC file for each grid. These were the values used to validate the 
Table 1 Table showing coding of bioclimatic variables

\begin{tabular}{|c|c|}
\hline Abbreviation & Description of bioclimatic variable \\
\hline $\mathrm{BIO1}$ & Annual Mean Temperature \\
\hline $\mathrm{BIO} 2$ & $\begin{array}{l}\text { Mean Diurnal Range (Mean of monthly (max } \\
\text { temp-min temp)) }\end{array}$ \\
\hline $\mathrm{BIO} 3$ & Isothermality (BIO2/BIO7) (* 100) \\
\hline $\mathrm{BIO} 4$ & Temperature Seasonality (standard deviation *100) \\
\hline $\mathrm{BIO5}$ & Max Temperature of Warmest Mont \\
\hline $\mathrm{BIO6}$ & Min Temperature of Coldest Month \\
\hline $\mathrm{BIO7}$ & Temperature Annual Range (BIO5-BIO6) \\
\hline $\mathrm{BlO8}$ & Mean Temperature of Wettest Quarter \\
\hline $\mathrm{BIO9}$ & Mean Temperature of Driest Quarter \\
\hline $\mathrm{BIO} 10$ & Mean Temperature of Warmest Quarter \\
\hline $\mathrm{BIO} 11$ & Mean Temperature of Coldest Quarter \\
\hline $\mathrm{BIO} 12$ & Annual Precipitation \\
\hline $\mathrm{BIO13}$ & Precipitation of Wettest Month \\
\hline $\mathrm{BIO} 14$ & Precipitation of Driest Month \\
\hline $\mathrm{BIO} 15$ & Precipitation Seasonality (Coefficient of Variation) \\
\hline $\mathrm{BIO} 16$ & Precipitation of Wettest Quarter \\
\hline $\mathrm{BIO} 17$ & Precipitation of Driest Quarter \\
\hline $\mathrm{BlO} 18$ & Precipitation of Warmest Quarter \\
\hline $\mathrm{BIO} 19$ & Precipitation of Coldest Quarter \\
\hline
\end{tabular}

accuracy performance and significance of the ecological niche models. The prediction models were all validated using the kappa statistics and receiver operator characteristics (ROC) area under curve (AUC). Interpretation of the acquired model performance values was done for kappa statistics and AUC values [19, 20].

\section{Results}

The predicted suitability maps generated from the three climate models have shown that climate change in Kenya might result to the spread of malaria vectors to new areas. The prediction results portrayed high likelihood of shift in some suitable habitats that could turn unsuitable, while new hotspots are likely to emerge. The BIOCLIM prediction with the three future climate models showed that the current endemic zones of the lake region and south coastal strip of the Indian Ocean will still remain suitable habitats but with a decline shift in spatial extent by the year 2020 , then start to expand by the years 2050-2080. Most current habitats are likely to still remain as viable malaria zones of diminished extent, or with continuous area gain into the neighboring non-endemic zones. Further change to climatic conditions was shown to be likely to trigger extremely new isolated hotspots that were not close to the current existing ecosystems. BIOCLIM prediction spatial models are shown in Fig. 3. The results for BIOCLIM True/
False are shown in Fig. 4. BIOCLIM True/False forecasting for malaria vector distribution was done to generate maps of two ecological zones with gray color denoting all the unsuitable areas and red color denoting the areas of excellent ecological conditions for malaria vectors to thrive. The predicted suitable malaria habitats resulted in an increase trend from the year 2020 to the year 2050 at higher magnitude in HADCM3 than in CSIRO projection, followed by further decrease in suitable ecology for malaria to survive by the year 2080 in both projections. However, the predicted ecological niche with suitable potential by 2080 will be lower than the area in the year 2020 as predicted with HADCM3 projection, while the reverse is true for prediction with CSIRO projection. CCCMA future climate prediction deviated from the trend observed in the two other climate projections.

BIOCLIM prediction with baseline climate (1950-2000) resulted into areas of different suitability as habitats for malaria vectors in Kenya. These ecological niches were analyzed, and the total area of non-suitability was found to be $285,830 \mathrm{~km}^{2}$. The total area found to be low in suitability was $273,055 \mathrm{~km}^{2}$. The ecological niche found to harbor medium potential occupied a total area of 63,917 $\mathrm{km}^{2}$ across Kenya. The area of high potential was 38, 939 $\mathrm{km}^{2}$, while area of very high suitability was found to be 11 , $861 \mathrm{~km}^{2}$. The total area that was found to be of excellent suitability was $6,044 \mathrm{~km}^{2}$.

When BIOCLIM prediction was done with climate data from HADCM3 projection by the year 2020, analysis of the ecological niches showed that the total area found to be non-suitable as malaria vector habitat will be $237,492 \mathrm{~km}^{2}$ by the year $2020,250,867 \mathrm{~km}^{2}$ by the year 2050 and 195 , $802 \mathrm{~km}^{2}$ by the year 2080 . The total area found to be low in suitability was $325,411 \mathrm{~km}^{2}$ by the year $2020,210,485 \mathrm{~km}^{2}$ by 2050 and $387,293 \mathrm{~km}^{2}$ by 2080 . The ecological niche found to harbor medium potential occupied a total area of $66,891 \mathrm{~km}^{2}$ by $2020,97,990 \mathrm{~km}^{2}$ by 2050 and $46,046 \mathrm{~km}^{2}$ by 2080 . The area of high suitability was predicted to be $43,215 \mathrm{~km}^{2}$ by $2020,58,728 \mathrm{~km}^{2}$ by 2050 and $39,846 \mathrm{~km}^{2}$ by 2080 , while area of very high suitability was predicted to be $13,167 \mathrm{~km}^{2}$ by $2020,56,033 \mathrm{~km}^{2}$ by 2050 and 18 , $582 \mathrm{~km}^{2}$ by 2080 . Excellent ecological niche will be 6545 $\mathrm{km}^{2}$ by 2020 , slightly more than that in the current climate condition, $18,618 \mathrm{~km}^{2}$ by 2050 and $5152 \mathrm{~km}^{2}$ by 2080 . The prediction by year 2080 using BIOCLIM model with HADCM3 projection was the lowest in excellent malaria vectors habitats.

BIOCLIM prediction with CCCMA future projection resulted into areas of different suitability as habitats for malaria vectors in Kenya. From the analyzed results, the total area of non-suitability will be $250,060 \mathrm{~km}^{2}$ by the year 2020, 235, $052 \mathrm{~km}^{2}$ by the year 2050 and 180, 984 $\mathrm{km}^{2}$ by the year 2080 . The total area of low suitability was 
Fig. 2 Flow diagram for correlative malaria vector distribution modeling

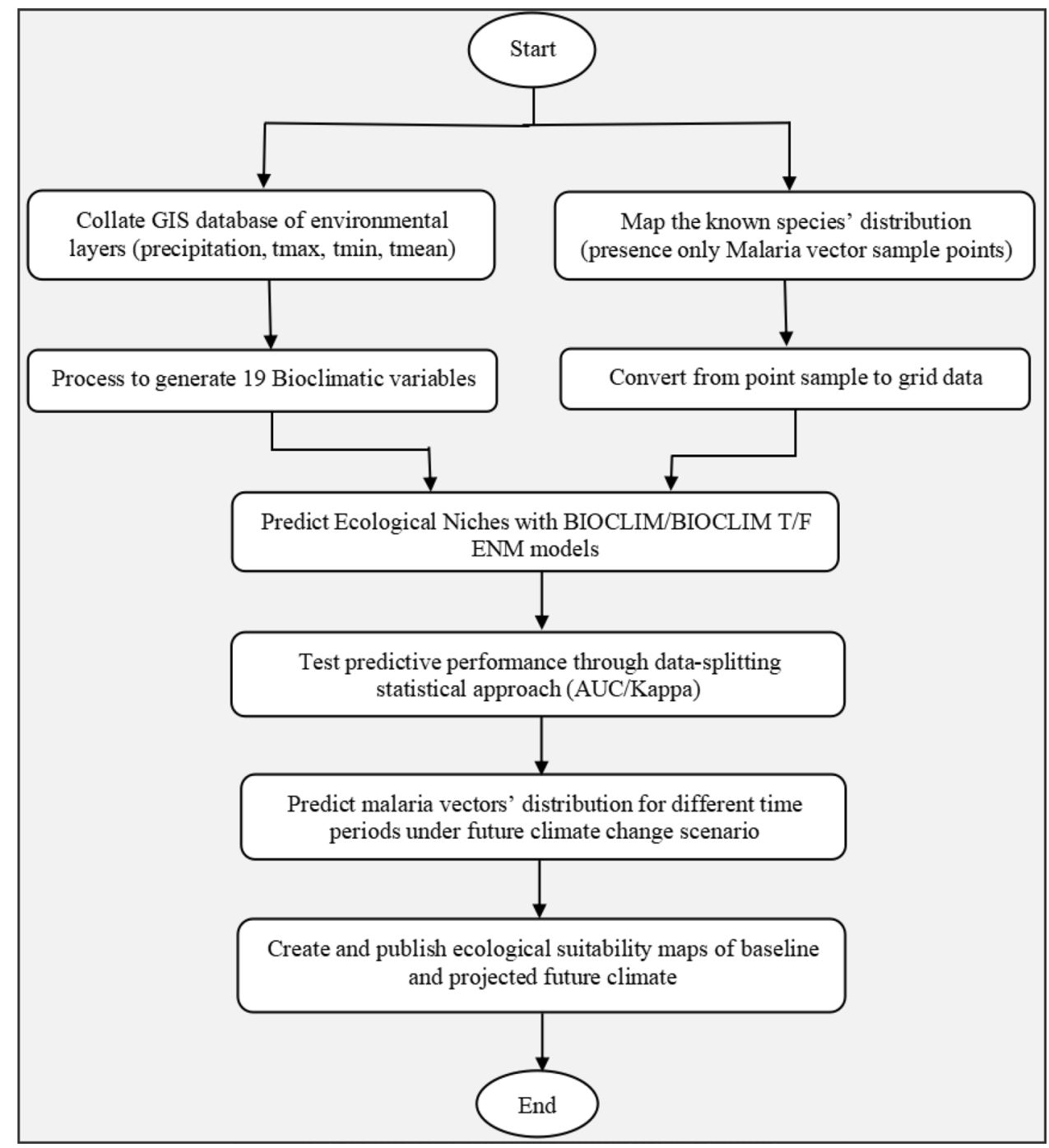

projected to be $323,901 \mathrm{~km}^{2}$ by the year $2020,355,460$ $\mathrm{km}^{2}$ by the year 2050 and $428,596 \mathrm{~km}^{2}$ by the year 2080 . The medium potential area was a total area of $65,794 \mathrm{~km}^{2}$ by the year $2020,51,655 \mathrm{~km} 2$ by 2050 and $34,241 \mathrm{~km} 2$ by 2080 . The area of high potential was $35,151 \mathrm{~km}^{2}$ by 2020 , $28,313 \mathrm{~km}^{2}$ by 2050 and $26,480 \mathrm{~km}^{2}$ by 2080 , while area of very high suitability was found to be $14,076 \mathrm{~km} 2$ by $2020,15,626 \mathrm{~km}^{2}$ by 2050 and $18,353 \mathrm{~km}^{2}$ by 2080 . The total area that was found to be of excellent suitability to potentially harbor malaria vectors was $3739 \mathrm{~km}^{2}$ by 2020, $6615 \mathrm{~km}^{2}$ and $4067 \mathrm{~km}^{2}$ by 2080.

When BIOCLIM prediction was done with climate data from CSIRO future projection, the six different ecological niches obtained were also recorded from delineated areas as shown in the spatial models for comparison. The categories were denoted as not suitable areas for malaria vectors' habitat (by 2020-219, $457 \mathrm{~km}^{2}$, by $2050-235,060 \mathrm{~km}^{2}$ and by $2080-222,813 \mathrm{~km}^{2}$ ); low suitability area (by $2020-379$, $921 \mathrm{~km}^{2}$, by $2050-353,833 \mathrm{~km}^{2}$ and by $2080-369,305$ $\mathrm{km}^{2}$ ); medium suitability area (by $2020-47,305 \mathrm{~km}^{2}$, by $2050-54,060 \mathrm{~km}^{2}$ and by $2080-43,770 \mathrm{~km}^{2}$ ); high suitability area (by $2020-27,219 \mathrm{~km}^{2}$, by $2050-31,539 \mathrm{~km}^{2}$ and by 2080-38, $056 \mathrm{~km}^{2}$ ); very high area (by $2020-5,662 \mathrm{~km}^{2}$, by $2050-14,790 \mathrm{~km}^{2}$ and by $2080-15,785 \mathrm{~km}^{2}$ ) and finally, excellent suitability areas (by $2020-3,157 \mathrm{~km}^{2}$, by $2050-3$, $439 \mathrm{~km}^{2}$ and by $2080-2,992 \mathrm{~km}^{2}$ ). Generally, potential excellent malaria vectors habitats were the least category with BIOCLIM model when all the three IPCC future climate projections were considered in prediction.

BIOCLIM True/False modeling resulted into two classes of either suitable or non-suitable malaria vectors habitats with the highest suitable habitat area of 227, 092 $\mathrm{km}^{2}$ obtained when prediction was done with HADCM3 future climate by the year 2050, and the least suitable habitat area of $80,060 \mathrm{~km}^{2}$ which was predicted with CCCMA projection by the year 2050. Predictions from HADCM 3 and CSIRO were of similar trend although at different magnitudes. In space and time, the predicted 


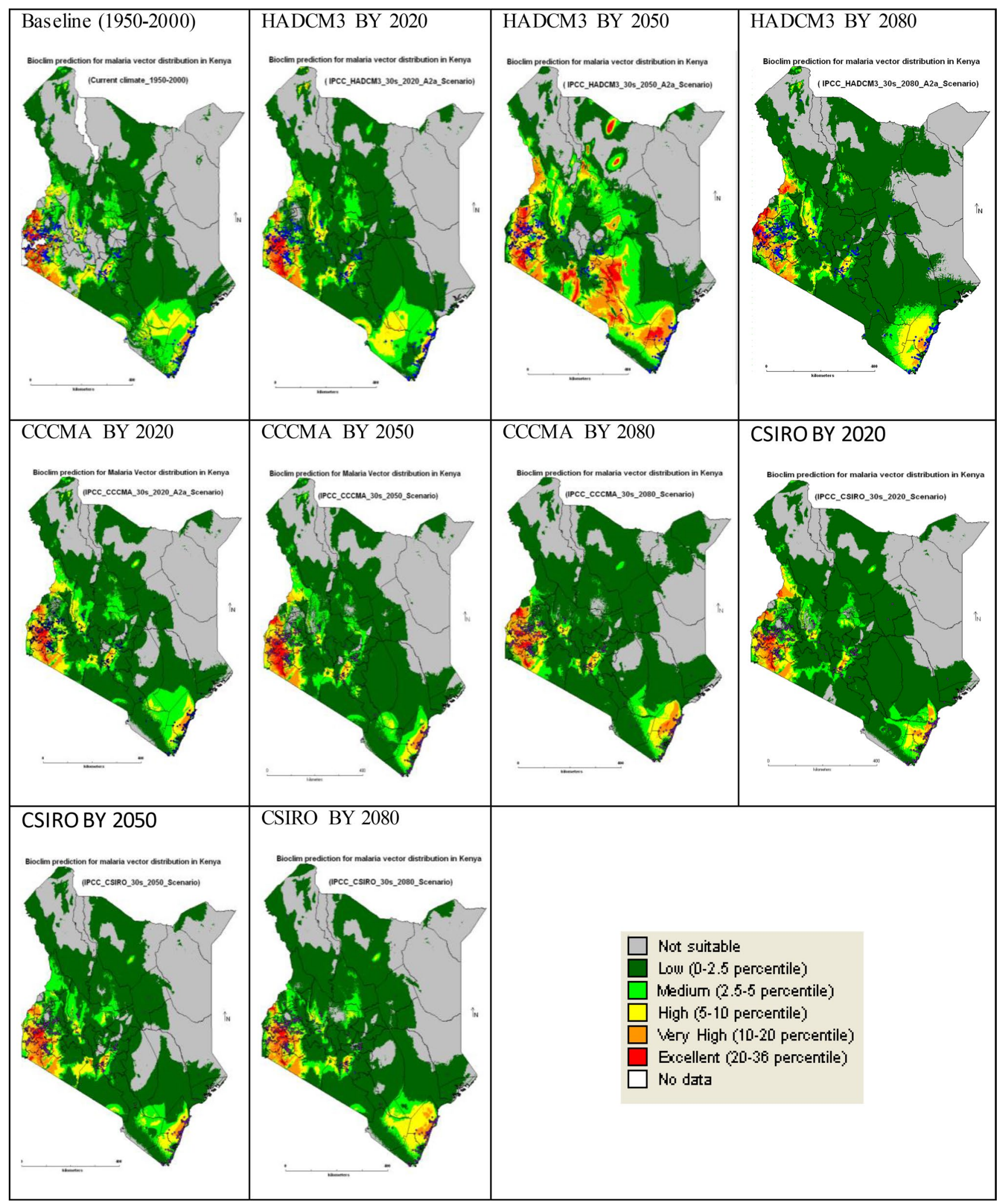

Fig. 3 BIOCLIM ENM spatial-temporal models for malaria vector distribution in Kenya for baseline climate (1950-2000) and IPCC projected 30 s future climate for HADCM3, CCCMA and CSIRO models by 2020, 2050 and 2080. Gray color denotes unsuitable habitats, while red is the areas of excellent suitability with the other classes shown as low, medium, high and very high suitability as described in the different colors 


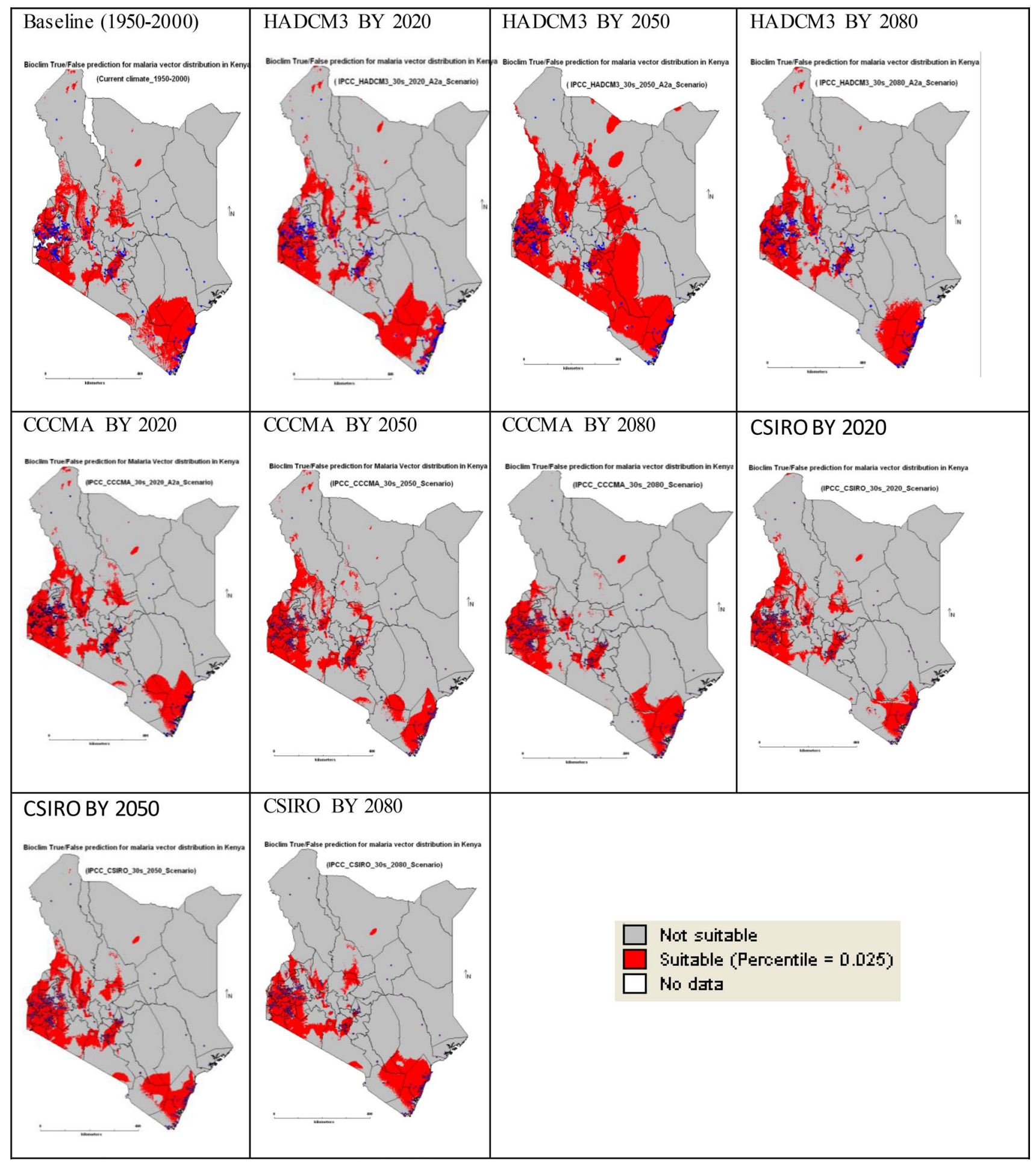

Fig. 4 BIOCLIM True/False ENM spatial-temporal models for malaria vector distribution in Kenya for baseline climate (19502000) and IPCC projected $30 \mathrm{~s}$ future climate for HADCM3, CCCMA and CSIRO models by 2020, 2050 and 2080. The suitable habitats are shown in red, while gray color denotes the unsuitable habitats

suitable habitats for malaria to survive by the year 2080 in both projections. However, the predicted ecological niche that might be suitable by 2080 will be lower than suitable habitats are likely to increase from the year 2020 to the year 2050 at higher magnitude in HADCM3 than in CSIRO projection, followed by further decrease in

\section{SN Applied Sciences}


the area in the year 2020 as predicted with HADCM3 projection, while the reverse is true for prediction with CSIRO projection. CCCMA future climate prediction deviated from the trend observed in the two other climate projections.

The area of suitable malaria ecology might decline from by the year 2020 prediction, through the year 2050 up to by the year 2080 . Some currently suitable malaria habitats are likely to become unsuitable, while new suitable endemic zones may emerge. These emerging ecologies can be broadening from the current niches, although new isolated hotspots might emerge even further away from the current endemic zones. For instance, predictions from HADCM3 by the year 2050 has shown wide spread of malaria spatial extents in counties like Narok, Kajiado, Kitui, Makueni, Machakos, Meru, Marsabit, Isiolo, Samburu, Baringo, West Pokot, Turkana and Mandera among a few others of lower extent that might have some emerging isolated malaria hotspots. Laikipia County might become unsuitable habitat for malaria vectors by the year 2050, and the case remains the same by the year 2080. The malaria burden is likely to shift from Laikipia to the neighboring counties of Baringo, Isiolo, Meru and Turkana. The county names are shown in "Appendix 1". Model validation results are shown in Fig. 5. The overall prediction performance for all the models was found to be within acceptable range. The lowest AUC value was 0.714 achieved from BIOCLIM True/False model with HADCM3 projection by 2020 , while the lowest kappa value was 0.427 also obtained from BIOCLIM True/False model with HADCM3 projection by 2020 . The highest AUC value was 0.869 achieved from BIOCLIM model with CCCMA projection by
2050, while the highest kappa value was 0.624 achieved from BIOCLIM model with CSIRO projection by 2080.

\subsection{Comparative analysis of BIOCLIM model prediction results}

Prediction of suitable habitats using BIOCLIM model resulted into six categorized classes where malaria vectors could be found. Representative bar graphs were generated for each BIOCLIM prediction with the three IPCC future projected climate scenarios. The areas for non-suitable ecological niches for malaria vectors to thrive in Kenya have been quantified based on baseline climate and IPCC future projections. The baseline climate (1950-2000) has the largest unsuitable area of $285,830 \mathrm{~km}^{2}$, while CCCMA by 2080 prediction has the least unsuitable area of 180,984 $\mathrm{km}^{2}$. Predicted non-suitable niches for HADCM3 and CSIRO projections have shown a similar trend of decrease in area from current to by the year 2020, with the area increasing by the year 2050, then further decrease by the year 2080. On the contrary, CCCMA projection deviates from this trend but portrays continuous decrease in ecological niche suitability from current climate through 2020 and 2050 up to by the year 2080. Comparative bar graphs of all the results from BIOCLIM predicted non-suitable and the suitable malaria vectors habitats of different magnitudes are shown in Fig. 6.

Further comparison of ecological niche areas from BIOCLIM model with HADCM3, CCCMA and CSIRO climate projections was done. Similar ecological niches are denoted with the same color for each climate scenario. The total area for the six ecological niches from the baseline climate was
Fig. 5 Model validation results (AUC/kappa) for BIOCLIM and BIOCLIM True/False prediction models with future climate projection models HADCM3, CCCMA and CSIRO by 2020, 2050 and 2080. BIOCLIM model had higher sensitivity than BIOCLIM True/False
MODEL VALIDATION RESULTS

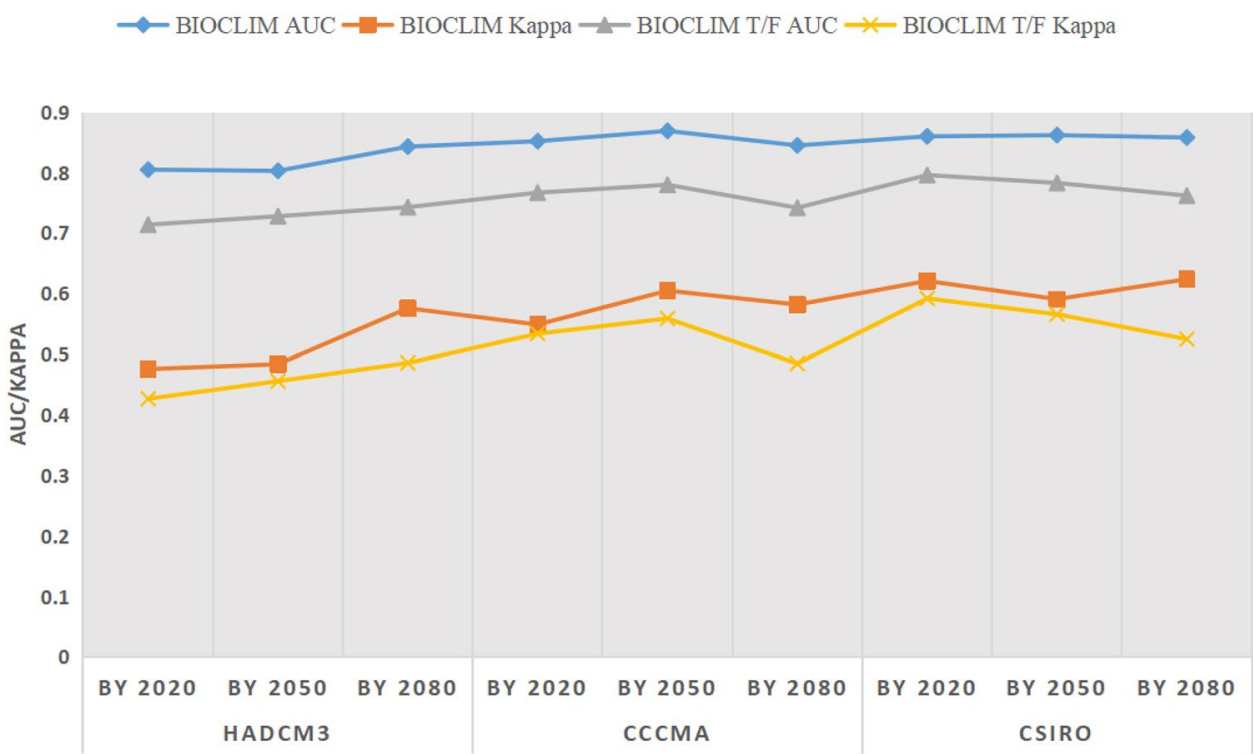

SN Applied Sciences A SPRINGER NATURE journat 


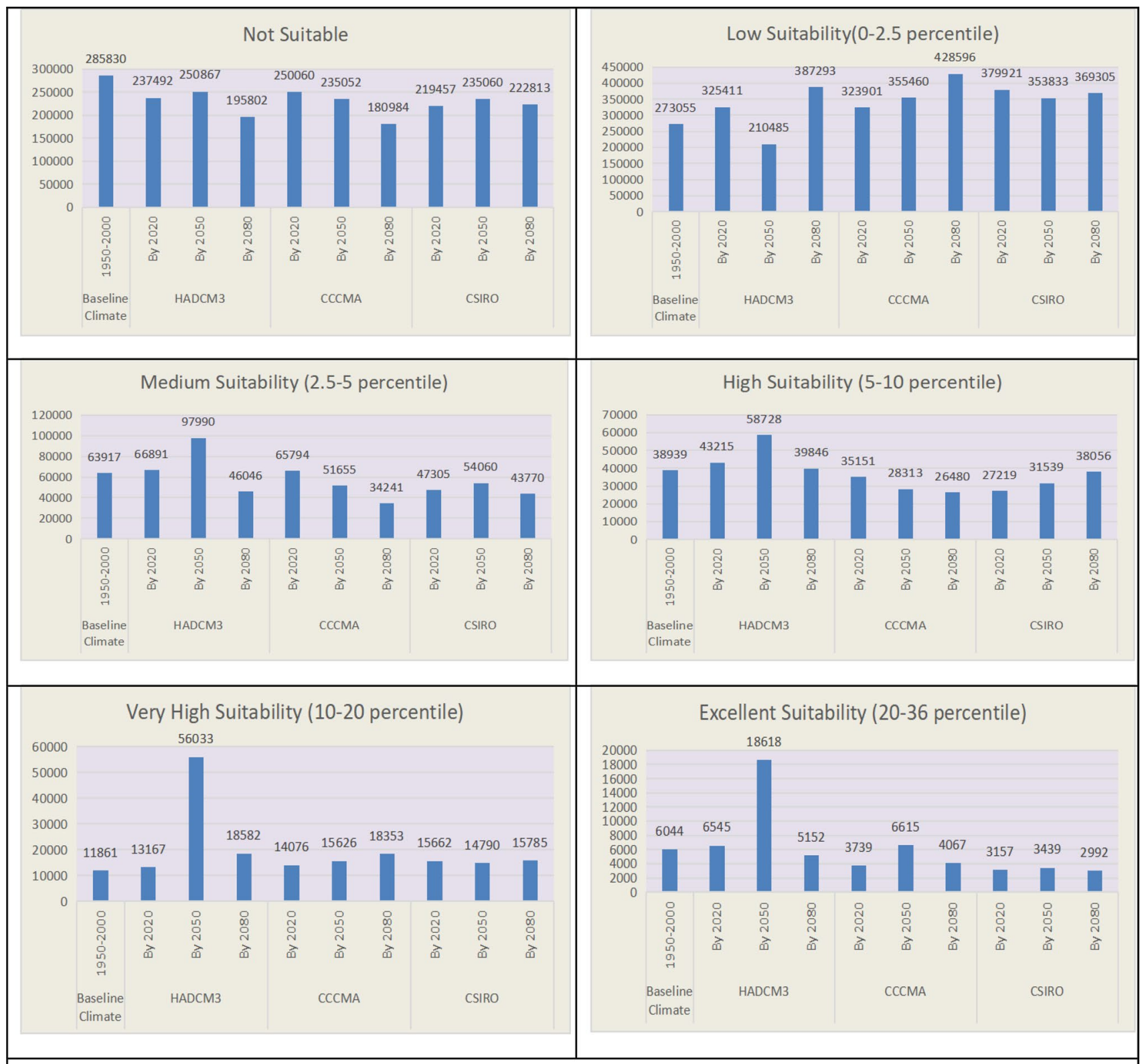

Fig. 6 BIOCLIM predicted areas of non-suitability, areas of low suitability, medium, high, very high and excellent suitability malaria vectors' habitats from baseline climate, HADCM3, CCCMA and CSIRO projections

found to vary slightly from the total area obtained in each future climate scenario. This can be attributed to the fact that ecological zones overlap with grid duplication thus variation in area summation as observed in the light blue bars. Interesting to note is the trend in which the viable malaria vector habitats are spreading from the baseline climate to projected climate conditions. The non-suitable ecological niche has diminished in all the predictions with IPCC projected climate as compared to the baseline climate prediction. There will be gain of area in the five suitable ecological niches cumulatively. These observations strongly demonstrated that climate change plays a major role in altering the suitable habitats for malaria vector to thrive in new areas in Kenya. The comparison of all the predicted ecological niches resulting from BIOCLIM ENM with the three IPCC future climate projections is shown in Fig. 7. The generated spatial models can be incorporated in any strategic plans to enhance malaria surveillance and combat malaria epidemics.

\subsection{Comparative analysis of BIOCLIM true/false model prediction results}

Ecological niche modeling using BIOCLIM True/False model resulted into two classes of either suitable or 


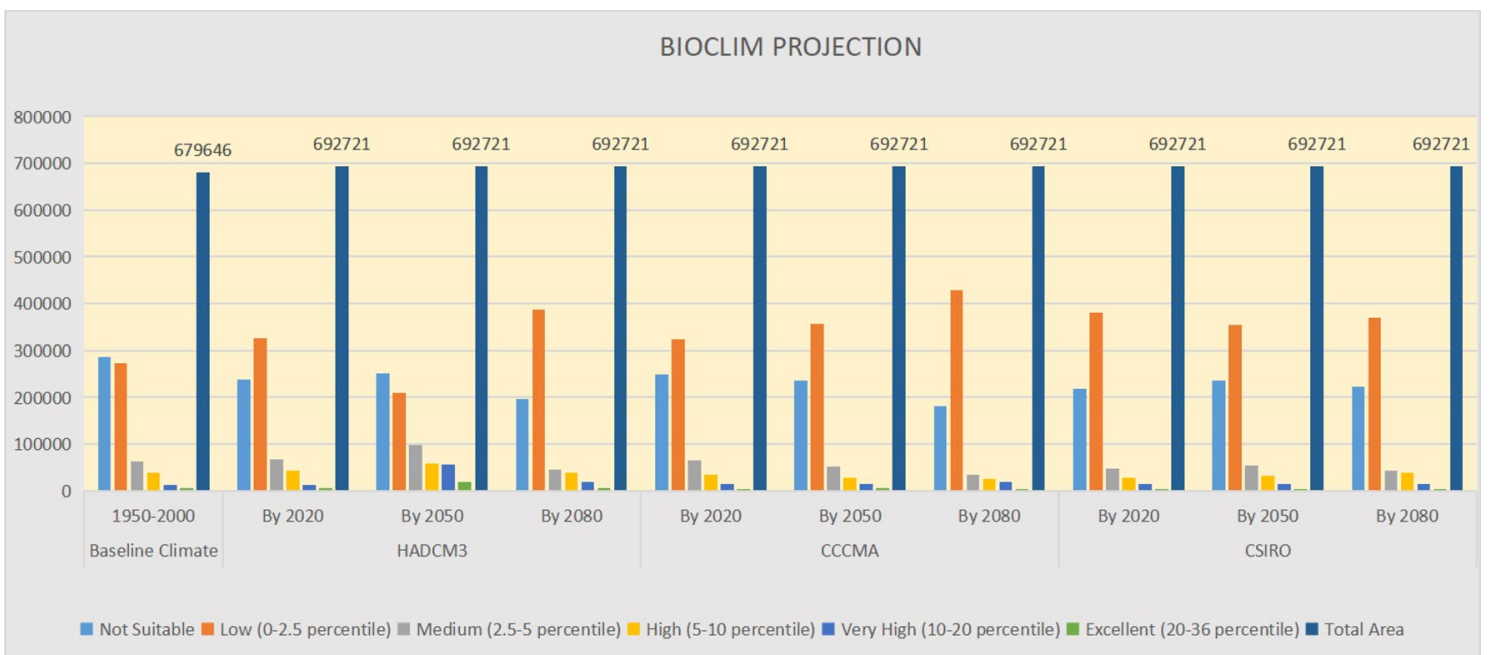

Fig. 7 Comparison of BIOCLIM predicted ecological niches for malaria vectors' habitats from baseline climate (1950-2000), HADCM3, CCCMA and CSIRO projections

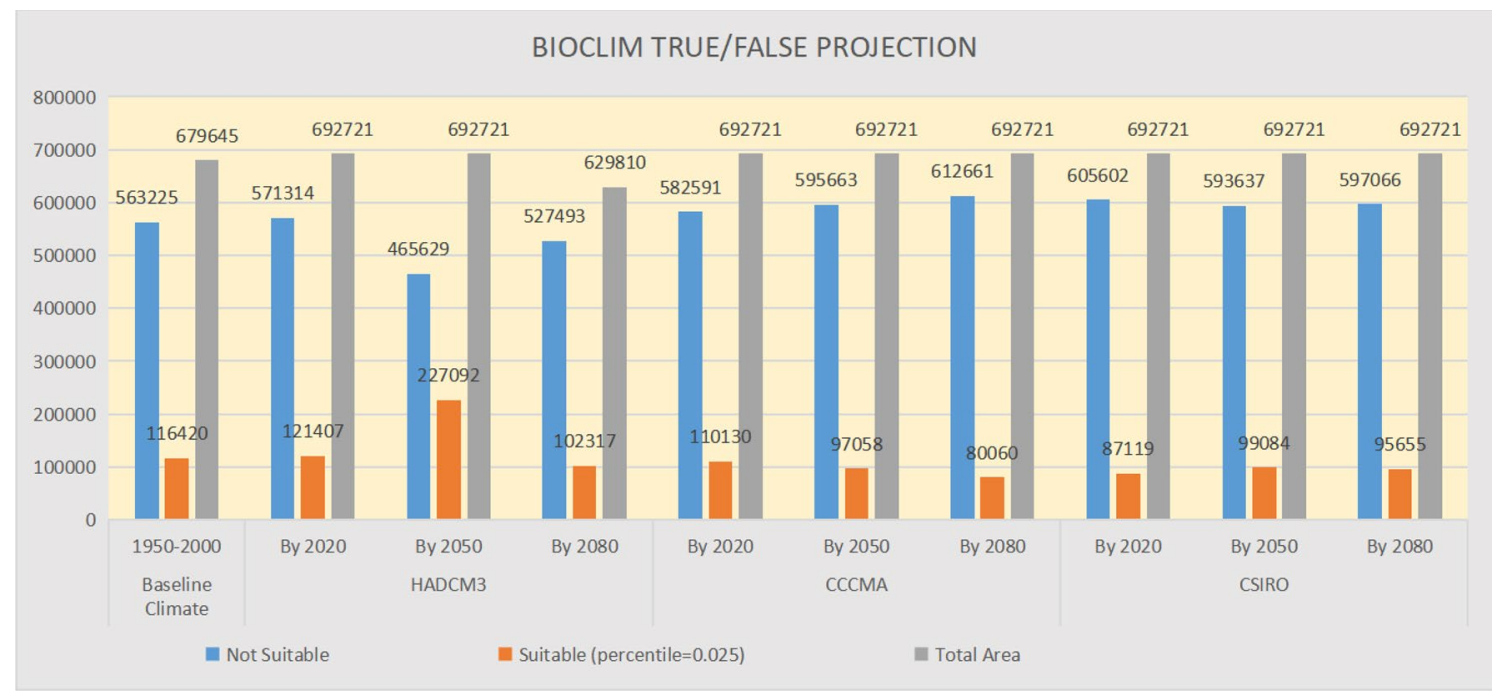

Fig. 8 Comparison of BIOCLIM True or False predicted ecological niches for malaria vectors' habitats from current climate (1950-2000), HADCM3, CCCMA and CSIRO projections 
non-suitable malaria vectors habitats. The highest suitable ecology was $227,092 \mathrm{~km}^{2}$ obtained when prediction was done with HADCM 3 future climate by the year 2050. The least suitable ecology was $80,060 \mathrm{~km}^{2}$ which was predicted with CCCMA projection by the year 2050. Ecological niche prediction from HADCM3 and CSIRO showed a similar trend although at different magnitudes. Figure 8 shows the comparison of BIOCLIM True/ False predicted ecological niches from baseline climate (1950-2000), HADCM3, CCCMA and CSIRO models.

\section{Discussion}

Climate change is postulated to alter the distribution and abundance of species which serve as vectors for pathogens and is thus expected to affect the transmission of infectious, vector-borne diseases such as malaria $[1,9]$. The future distribution of malaria in Africa is likely to be much more dependent on environmental conditions than the current distribution due to the effectiveness of indoor and therapeutic anti-malarial interventions, such as insecticide-treated nets (ITNs), indoor residual spraying for mosquitoes (IRS), artemisinin combination therapy (ACT), and intermittent presumptive treatment (IPT). However, adoption of multiple ENM methods to study disease vector distributions is slow relative to the general ENM literature [21]. There is a wealth of evidence that recent climate change has already affected pathogen-vector-host systems, in particular over-temperate, peri-Arctic and Artic areas and high-altitude regions in the tropics [22]. Correlative models articulate on the understanding of parameters that are the most important explanatory variables in the climate enhanced suitability for particular disease vector or a host in specific geographical extent. Further, mechanistic models that describe the biological/ecological processes of the transmission are needed [23].

BIOCLIM and BIOCLIM True/False models were employed to determine the possible future malaria vector prevalence by ecological niches. Future prediction for the prevalence and distribution of the malaria vectors was done with model data from HADCM3, CCCMA and SCIRO IPCC future climatic projections under A2a scenario in Kenya. Climate envelope prediction models were generated for the current climate and projections done to depict how the vectors are likely to be distributed by the years 2020, 2050 and 2080 under climate change impact (Figs. 3, 4). Comparative ecological area analysis for all the predictions was quantified (Figs. 6, 7, 8]. Spatial-temporal models for risk management of climate change-induced malaria vectors surge in new hotspots were generated for comparative analyses and visualization to guide policy and any effort that involves adaptation and mitigation decisions with implications for future generations, economies and environments.

Some currently suitable malaria habitats might become unsuitable, with new suitable hotspots likely to emerge. These emerging habitats will be likely to extend from the current niches, with possible emergence of completely new isolated hotspots of random distribution. The generated spatial-temporal models depicted how the vectors are likely to be distributed by the years 2020, 2050 and 2080 under the influence of climate change/variability. The prediction results portrayed high likelihood of shift in some suitable habitats that could turn unsuitable, while new hotspots are likely to emerge. The BIOCLIM prediction with the three future climate models showed that the current excellent habitats of the lake region and south coastal strip of the Indian ocean will remain suitable but with a decline shift in spatial extent by the year 2020, then start to expand by the years 2050 to 2080 . The results have some resonance with the findings in Macharia et al. [5]. In order to provide recommendations, based on evidences that can be transformed into policies, the impact of climate change has to be investigated at the very specific and local scale [24]. 


\section{Conclusion}

The study aim was to employ comparative spatial-temporal analysis using climate envelope modeling to predict and quantify the possible surge of suitable malaria vectors habitats in new hotspots in Kenya. Ecological niche modeling was applied to spatially establish the effect of climate as an explanatory variable in main malaria vector distribution, hence malaria prevalence in new hot spots. Predictive modeling was done to investigate the spatial-temporal vector distribution under different IPCC future climate projections. The period from 1950 to 2000 was treated as the baseline climate scenario to explain where malaria vectors are existing as per vector spatial presence data and the prediction of the ecological niches where the vectors would also be found to thrive.

The research study provides crucial information of great utility and importance. In order to safeguard the populations, there is need to create awareness for preparedness and early risk management in the current non-endemic zones that have the potential of becoming future malaria vectors' hotspots. The global concerted effort to increase resilience among the indigenous communities and promote the participation of the local population through integration of indigenous knowledge to scientific methods should be scaled up so as to reduce vulnerability and enhance adaptive capacity in malaria endemic zones. Evidence-based scientific research on future malaria hotspots should be utilized to guide interventions on vector control so as to curb the epidemic and enhance resilience to climate change/ variability-related vulnerabilities.

In conclusion, intervention measures for malaria catastrophes in Kenya should be well-planned to capture the future suitable malaria habitats and emerging hotspots in all the newly predicted regions to ensure equitable resource deployment. Policy on adaptation and mitigation strategies should be well-formulated and communicated to the unsuspecting populations residing in the currently malaria-free zones found to harbor the potential for future epidemics. Henceforth, recommendation is to upscale modeling with current actual malaria surveillance data to validate model predictions by the year 2020, thus providing a new baseline of current data. The research study is of great significance as it informs policy on malaria vectors control in Kenya. Scientific research evidence coupled with expanded targeted interventions has unrivaled potential in control and containment of malaria epidemic in endemic and non-endemic zones with likely potential for future malaria prevalence.

Authors' contributions All the aspects of data collection, research study design and manuscript compilation was conducted by the author.

Funding The research was accomplished through funding from Kenya National Council for Science and Technology, Ministry of Higher Education Science, Technology and Innovation Grant Fund (ST\&l); Grant File No. NCST/ACC/003/005/154/4th CALL PhD.

Availability of data and material The sources of data and materials used to accomplish the research study are included within the article.

Code availability This is not applicable as freeware DIVA-GIS software was used to perform predictive modeling.

\section{Declarations}

Conflict of interest The author declares that there is no conflict of interest in this original research study. 


\section{Appendix 1}

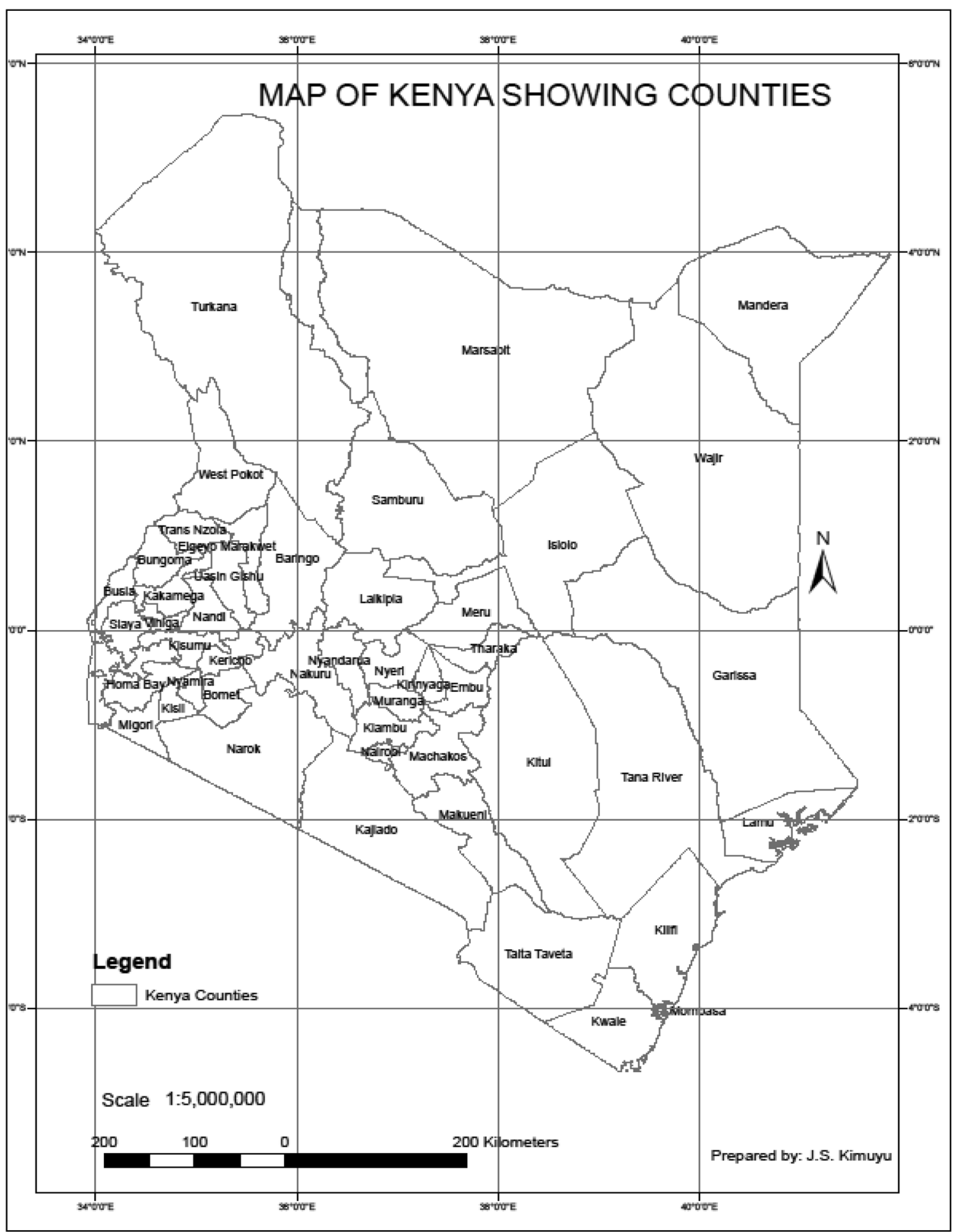


Open Access This article is licensed under a Creative Commons Attribution 4.0 International License, which permits use, sharing, adaptation, distribution and reproduction in any medium or format, as long as you give appropriate credit to the original author(s) and the source, provide a link to the Creative Commons licence, and indicate if changes were made. The images or other third party material in this article are included in the article's Creative Commons licence, unless indicated otherwise in a credit line to the material. If material is not included in the article's Creative Commons licence and your intended use is not permitted by statutory regulation or exceeds the permitted use, you will need to obtain permission directly from the copyright holder. To view a copy of this licence, visit http://creativecommons. org/licenses/by/4.0/.

\section{References}

1. Kimuyu JS, Muthama NJ, Musyoka SM (2017) Ecological niche modeling for spatial-temporal quantification of the changing dynamics of malaria vector distribution in Kenya under climate change forcing. IJSTR 6(07):319-332

2. Snow RW, Gouws E, Omumbo J, Rapuoda B, Craig MH, Tanser FC et al (1998) Models to predict the intensity of Plasmodium falciparum transmission: application to the burden of disease in Kenya. Trans R Soc Trop Med Hyg 92:601-606

3. Noor AM, Gething PW, Alegana VA, Patil AP, Hay SI, Muchiri E et al (2009) The risks of malaria infection in Kenya in 2009. BMC Infect Dis 9:180

4. Gething PW, Van Boeckel TP, Smith DL, Guerra CA, Patil AP, Snow RW et al (2011) Modelling the global constraints of temperature on transmission of Plasmodium falciparum and $P$. vivax. Parasites Vectors 4:92

5. Macharia PM, Giorgi E, Noor AM et al (2018) Spatio-temporal analysis of Plasmodium falciparum prevalence to understand the past and chart the future of malaria control in Kenya. Malar J 17:340. https://doi.org/10.1186/s12936-018-2489-9

6. Platt A, Obala AA, MacIntyre C, Otsyula B, O'Meara WP (2018) Dynamic malaria hotspots in an open cohort in western Kenya. Sci Rep 8:647. https://doi.org/10.1038/s41598-017-13801-6

7. Alahmed AM, Naeem M, Kheir SM, Sallam FM (2015) Ecological distribution modeling of two malaria mosquito vectors using geographical information system in Al-Baha Province, Kingdom of Saudi Arabia. Pak J Zool 47(6):1797-1806

8. Chang KS, Yoo D-H, Ju YR, Lee WG, Roh JY, Kim H-C, Klein TA, Shin E-H (2016) Distribution of malaria vectors and incidence of vivax malaria at Korean army installations near the demilitarized zone, Republic of Korea. Malaria J 15:259. https://doi.org/ 10.1186/s12936-016-1301-y

9. Karypidou MC, Almpanidou V, Tompkin AM et al (2020) Projected shifts in the distribution of malaria vectors due to climate change. Clim Change. https://doi.org/10.1007/ s10584-020-02926-9

10. Obsomer V, Defourny P, Coosemans M (2012) Predicted distribution of major malaria vectors belonging to the anopheles dirus complex in Asia: ecological niche and environmental influences.
PLoS ONE 7(11):e50475. https://doi.org/10.1371/journal.pone. 0050475

11. Ren Z, Wang D, Ma A, Hwang J, Bennett A, Sturrock HJW, Fan J, Zhang W, Yang D, Feng X, Xia Z, Xiao-Nong Z, Wang J (2016) Predicting malaria vector distribution under climate change scenarios in China: challenges for malaria elimination. Sci Rep 6:20604. https://doi.org/10.1038/srep20604

12. Sallam MF, Xue R-D, Pereira RM, Phillip G, Koehler PG (2016) Ecological niche modeling of mosquito vectors of West Nile virus in St John's County, Florida, USA. Parasites Vectors 9:371. https:// doi.org/10.1186/s13071-016-1646-7

13. Song Y, Ge Y, Wang J, Ren Z, Liao Y, Peng J (2016) Malaria J 15:345. https://doi.org/10.1186/s12936-016-1395-2

14. Ministry of Health (MOH) [Kenya] (2014) The Kenya Malaria Strategy 2009-2018 (Revised 2014). Nairobi, Kenya: Ministry of Public Health and Sanitation

15. World Health Organization (2020) Strategic advisory group on malaria eradication. Malaria eradication: benefits, future scenarios and feasibility. A report of the strategic advisory group on malaria eradication. Geneva: Licence: CC BY-NC-SA 3.0 IGO.

16. IPCC, (2014) Climate Change 2014: impacts, adaptation, and vulnerability. Part A: global and sectoral aspects. Contribution of Working Group II to the Fifth Assessment Report of the 12 Intergovernmental Panel on Climate Change [Field, C.B., V.R. Barros, D.J. Dokken, K.J. Mach, M.D. Mastrandrea, T.E. Bilir, M. Chatterjee, K.L. Ebi, Y.O. Estrada, R.C. Genova, B. Girma, E.S. Kissel, A.N. Levy, S. MacCracken, P.R. Mastrandrea, and L.L. White (eds.)]. Cambridge University Press, Cambridge, United Kingdom and New York, NY, USA, $1132 \mathrm{pp}$

17. Okara RM, Sinka ME, Minakawa N, Mbogo CM, Hay SI, Snow RW (2010) Distribution of the main malaria vectors in Kenya. Malar J 9:69. https://doi.org/10.1186/1475-2875-9-69

18. Hijmans RJ, Cameron SE, Parra JL, Jones PG, Jarvis A (2005) Very high resolution interpolated climate surfaces for global land areas. Int J Climatol 25(15):1965-1978. https://doi.org/10.1002/ joc. 1276

19. Geary RC (1954) The contiguity ratio and statistical mapping. Inc Stat 5:115-141

20. Moran PAP (1948) The interpretation of statistical maps. J R Stat Soc B 10:243-251

21. Drake JM, Beier JC (2014) Ecological niche and potential distribution of Anopheles arabiensis in Africa in 2050. Malar J 13:213. https://doi.org/10.1186/1475-2875-13-213

22. Caminade C, McIntyre KM, Jones AE (2018) Impact of recent and future climate change on vector-borne

23. Tjaden NB, Caminade C, Beierkuhnlein C, Thomas SM (2018) Mosquito-borne diseases: advances in modelling climatechange impacts. Trends Parasitol 34(3):227-245. https://doi. org/10.1016/j.pt.2017.11.006

24. Fouque F, Reeder JC (2019) Impact of past and on-going changes on climate and weather on vector-borne diseases transmission: a look at the evidence. Infect Dis Poverty 8:51. https://doi.org/10.1186/s40249-019-0565-1

Publisher's Note Springer Nature remains neutral with regard to jurisdictional claims in published maps and institutional affiliations. 